\title{
MINIMAL DISPLACEMENT OF POINTS UNDER HOLOMORPHIC MAPPINGS AND FIXED POINT PROPERTIES FOR UNIONS OF CONVEX SETS
}

\author{
TADEUSZ KUCZUMOW, SIMEON REICH, AND ADAM STACHURA
}

\begin{abstract}
Let $D$ be an open convex bounded subset of a complex Banach space $(X,\|\cdot\|)$, and let $C$ be the union of a finite number of closed convex sets lying strictly inside $D$. Using the Kuratowski measure of noncompactness with respect to the Kobayashi distance in $D$, we first show that if $f: D \rightarrow D$ is a holomorphic mapping which leaves $C$ invariant, and if the Lefschetz number $\lambda\left(f_{\mid C}\right) \neq 0$, then $\inf \{\|x-f(x)\|: x \in C\}=0$. We then deduce several new fixed point theorems for holomorphic and nonexpansive mappings.
\end{abstract}

\section{INTRODUCTION}

Let $D$ be an open convex bounded subset of a complex Banach space $(X,\|\cdot\|)$, and let $k_{D}$ denote the Kobayashi distance in $D$. Let $C$ be the union of a finite number of closed convex sets lying strictly inside $D$. Our first result is that if $f: D \rightarrow D$ is a holomorphic mapping which leaves $C$ invariant, and if the Lefschetz number $\lambda\left(f_{\mid C}\right) \neq 0$, then

$$
\inf \{\|x-f(x)\|: x \in C\}=0 .
$$

In the special case when $D=B^{n}$, the Cartesian product of $n$ unit open balls of a complex Hilbert space $H$, it then follows that if $f: B^{n} \rightarrow B^{n}$ is holomorphic, $C=\bigcup_{i=1}^{m} C_{i}$ is $f$-invariant, each $C_{i}$ is a closed convex $k_{D}$-bounded subset of $B^{n}$, and

$$
C_{i} \cap C_{j} \neq \varnothing \Leftrightarrow|i-j| \leq 1
$$

for all $1 \leq i, j \leq m$, then $f$ has a fixed point in $C$.

Our minimal displacement result is inspired by a theorem of Furi and Martelli [11] concerning $k$-set-contractions in normed spaces. However, their theorem does not yield the estimate (1) because $f$ is only norm-Lipschitzian on $C$ (and not norm-nonexpansive). Therefore we use the Kuratowski measure of noncompactness with respect to the Kobayashi distance $k_{D}$ and not with respect to the norm as in [11]. This new approach to holomorphic mappings and the metric character of our arguments show that the above results can, in fact, be

Received by the editors February 17, 1992.

1991 Mathematics Subject Classification. Primary 32H15, 47H09, 47H10.

Key words and phrases. Fixed point, holomorphic mapping, Kobayashi distance, measure of noncompactness, minimal displacement, nonexpansive mapping.

The third author was supported by the KBN under Grant 212609101 . 
extended to the family of all $k_{D}$-nonexpansive self-mapping of $C$. Moreover, the method of proof of our fixed point theorem for holomorphic mappings also leads to the following new fixed point theorem for nonexpansive mappings in Banach spaces: If $C_{1}, C_{2}, \ldots, C_{m}$ are $m$ nonempty weakly compact convex subsets of a Banach space, each $C_{i}$ has a normal structure, and (2) is satisfied, then every nonexpansive self-mapping of $C=\bigcup_{i=1}^{m} C_{i}$ has a fixed point. The case $m=1$ of this result is Kirk's classical theorem [21], while the case $m=2$ is a recent remarkable result of Stachura [46]. Several related results are also included.

\section{The Kobayashi distance AND the KURATOWSKI MEASURE OF NONCOMPACTNESS}

Let $(X,\|\cdot\|)$ be a complex Banach space and $D$ a nonempty, open, convex, and bounded subset of $X$. Let $k_{D}$ denote the Kobayashi distance in $D$ (see $[10,15,19,22])$. Directly from the definition of $k_{D}$ we obtain

$$
k_{D}(t x+(1-t) y, t w+(1-t) z) \leq \max \left[k_{D}(x, w), k_{D}(y, z)\right]
$$

for all $0 \leq t \leq 1$ and every choice of $x, y, w, z$ from $D$ [31]. This implies that the balls in $\left(D, k_{D}\right)$ are convex subsets of $X$.

We say that a subset $C$ of $D$ lies strictly inside $D(C \Subset D)$ if $\operatorname{dist}(C, \partial D)>$ 0 , or equivalently, if there exists a positive $R$ such that the ball (in $X,\|\cdot\|)$ ) $B(x, R) \subset D$ for all $x$ in $C$. A mapping $f: D \rightarrow D$ is said to map $D$ strictly inside $D$ if $f(D)$ lies strictly inside $D$.

If $C \Subset D$ is nonempty and convex, then the restriction of $k_{D}$ to $C \times C$ is Lipschitz-equivalent to the norm $\|\cdot\|[10,15,19]$. This means that there exist constants $L_{1}(C) \geq 1$ and $L_{2}(C)$ such that

$$
k_{D}(x, y) \leq L_{1}(C)\|x-y\| \leq L_{2}(C) k_{D}(x, y)
$$

for every pair of points $x, y \in C$. This implies that for every nonempty set $C \Subset D$ the inequalities (4) are valid. Using (3) and (4) we also see that

$$
\operatorname{diam}_{k_{D}}(\operatorname{clco} C)=\operatorname{diam}_{k_{D}}(C)
$$

for every $C \Subset D$, where clco $C$ denotes the closed convex hull of $C$.

Since the Kobayashi distance is the integrated form of the Kobayashi metric [10], the following theorem is valid.

Theorem 1 [10]. Let $f: D \rightarrow D$ be a holomorphic mapping which maps $D$ strictly inside $D$. Then $f$ is a strict contraction in $\left(D, k_{D}\right)$ with Lipschitz constant $K=1 /(1+s)$, where $s=R / 2 M, M=\operatorname{diam}(D)$, and $R=\operatorname{dist}(f(D), \partial D)$.

Remark. Theorem 1 is also valid for the Carathéodory integrated form $\tilde{c}_{D}$ of the Carathéodory metric [7, 15, 19].

Theorem 1 yields the following two corollaries.

Corollary 1. For each $x \in D$ and $0 \leq t<1$ the mapping $D \ni y \rightarrow(1-t) x+t y \in$ $D$ is a $k_{D}$-contraction with constant

$$
K=\frac{2[\operatorname{diam}(D)]}{2[\operatorname{diam}(D)]+(1-t) R},
$$

where $R$ is the radius of an open ball $B(x, R)($ in $(X,\|\cdot\|))$, which lies in $D$. 
Since every holomorphic mapping $f: D \rightarrow D$ is $k_{D}$-nonexpansive $[10,15$, 19], i.e.,

$$
k_{D}(f(x), f(y)) \leq k_{D}(x, y)
$$

for all $x, y \in D$, we also obtain the following result.

Corollary 2. For each fixed $x \in D$ and $0 \leq t<1$, and each holomorphic mapping $f: D \rightarrow D$, the mapping $D \ni y \rightarrow(1-t) x+t f(y) \in D$ is a $k_{D^{-}}$ contraction with a constant given by (6).

Now we discuss the Kuratowski measure of noncompactness in $\left(D, k_{D}\right)$. Let $C \Subset D$ be a nonempty subset of $D$. The number

$\alpha(C)=\inf \left\{d: C\right.$ has a finite covering of subsets of $D$ with $\operatorname{diam}_{k_{D}}$ less than $\left.d\right\}$ is called the Kuratowski measure of noncompactness of $C$ [32]. The measure $\alpha$ shares the following properties with all measures of noncompactness [2]:

(1) $\alpha(C) \leq \operatorname{diam}_{k_{D}}(C)$,

(2) if $C_{1} \subset C_{2}$, then $\alpha\left(C_{1}\right) \leq \alpha\left(C_{2}\right)$,

(3) $\alpha\left(C_{1} \cup C_{2}\right)=\max \left\{\alpha\left(C_{1}\right), \alpha\left(C_{2}\right)\right\}$

for all subsets $C, C_{1}, C_{2}$ of $D$ that lie strictly inside $D$.

We also need several other properties of $\alpha$.

Lemma 1. Let $C \Subset D$ be nonempty. Then

(a) $\alpha(C)=0$ if and only if the closure $\operatorname{cl}(C)$ of $C$ is compact;

(b) $\alpha(C)=\alpha(\operatorname{clco} C)$;

(c) if $C_{1} \Subset D$ and $C_{2} \Subset D$ are nonempty, $\alpha\left(C_{1}\right)=0$ and $0 \leq t<1$, then

$$
\alpha\left((1-t) C_{1}+t C_{2}\right) \leq K \alpha\left(C_{2}\right),
$$

where $K$ is given in (6) with $R=\operatorname{dist}\left(C_{1}, \partial D\right)$.

Proof. (a) It is sufficient to apply the inequalities (4).

(b) If $E$ is a subset of $X$ and $\eta>0$ we denote the set $\{y \in X: \operatorname{dist}(y, E)<$ $\eta\}$ by $E_{\eta}$.

The set clco $C$ also lies strictly inside $D$. Let $R=\operatorname{dist}(C, \partial D)$ and let $\varepsilon$ be an arbitrary positive number less than $\frac{1}{2} R$. For this $\varepsilon$ there is a covering $\left\{C_{1}, C_{2}, \ldots, C_{m}\right\}$ of $C$ such that $C_{1}, \ldots, C_{m}$ are closed and convex,

$$
\operatorname{diam}_{k_{D}} C_{i}<\alpha(C)+\frac{1}{3} \varepsilon, \quad \text { and } \operatorname{dist}\left(C_{i}, \partial_{D}\right) \geq R
$$

for $i=1,2, \ldots, m$. Next we choose $n$ satisfying

$$
L_{1}\left((\operatorname{clco} C)_{R / 2}\right) \frac{2(m-1)}{n} \sup _{x \in D}\|x\|<\frac{\varepsilon}{3},
$$

where $L_{1}\left((\text { clco } C)_{R / 2}\right)=L_{1}$ is the constant in (4) with $C$ replaced by $(\text { clco } C)_{R / 2}$. Now consider the family of sets

$$
\left(\frac{k_{1}}{n} C_{1}+\frac{k_{2}}{n} C_{2}+\cdots+\frac{k_{m}}{n} C_{m}\right)_{\varepsilon / 3 L_{1}},
$$

where $k_{1}, \ldots, k_{m}$ are nonnegative integers and $k_{1}+\cdots+k_{m}=n$. It is easy to observe that this family is a covering of clco $C$ and that the $k_{D}$-diameter of each set of this covering is less than $\alpha(C)+\varepsilon$. Hence $\alpha($ clco $C)=\alpha(C)$. 
(c) Let $\varepsilon$ be a positive number. For this $\varepsilon$ there exist points $\alpha_{1}, \ldots, \alpha_{m}$ in $C_{1}$ such that the $k_{D}$-balls $A_{1}, \ldots, A_{m}$ centered at $a_{i} \in C_{1}$ (respectively) with $k_{D}$-radii equal to $\varepsilon$ form a covering of $C_{1}$. Let $B_{1}, \ldots, B_{n}$ be a covering of $C_{2}$ with $\operatorname{diam}_{k_{D}} B_{j}<\alpha\left(C_{2}\right)+\varepsilon$ for $j=1, \ldots, n$. Then

$$
(1-t) C_{1}+t C_{2} \subset \bigcup_{i=1}^{m} \bigcup_{j=1}^{n}\left[(1-t) A_{i}+t B_{j}\right] .
$$

Applying (3) and Corollary 1 we obtain

$$
\begin{aligned}
& \operatorname{diam}_{k_{D}}\left[(1-t) A_{i}+t B_{j}\right] \\
& =\sup \left\{k_{D}\left((1-t) a^{\prime}+t b^{\prime},(1-t) a^{\prime \prime}+t b^{\prime \prime}\right): a^{\prime}, a^{\prime \prime} \in A_{i} ; b^{\prime}, b^{\prime \prime} \in B_{j}\right\} \\
& \leq \sup \left\{k_{D}\left((1-t) a^{\prime}+t b^{\prime},(1-t) a_{i}+t b^{\prime}\right)\right. \\
& \quad \quad+k_{D}\left((1-t) a_{i}+t b^{\prime},(1-t) a_{i}+t b^{\prime \prime}\right) \\
& \left.\quad \quad+k_{D}\left((1-t) a_{i}+t b^{\prime \prime},(1-t) a^{\prime \prime}+t b^{\prime \prime}\right): a^{\prime}, a^{\prime \prime} \in A_{i} ; b^{\prime}, b^{\prime \prime} \in B_{j}\right\} \\
& \leq \sup \left\{k_{D}\left(a^{\prime}, a_{i}\right)+K k_{D}\left(b^{\prime}, b^{\prime \prime}\right)+k_{D}\left(a_{i}, a^{\prime \prime}\right): a^{\prime}, a^{\prime \prime} \in A_{i} ; b^{\prime}, b^{\prime \prime} \in B_{j}\right\} \\
& \leq(2+K) \varepsilon+K \alpha\left(C_{2}\right) .
\end{aligned}
$$

Hence

$$
\alpha\left((1-t) C_{1}+t C_{2}\right) \leq K \alpha\left(C_{2}\right)
$$

\section{Minimal DisPlacement}

We say that a finite family $\mathscr{C}$ of nonempty closed convex sets lying strictly inside $D$ is complete if $C_{1} \cap C_{2} \in \mathscr{C}$ whenever $C_{1}, C_{2} \in \mathscr{C}$ and $C_{1} \cap C_{2} \neq \varnothing$ [11]. Every finite family of nonempty closed convex sets lying strictly inside $D$ is a subfamily of a complete family. The symbol $|\mathscr{C}|$ will denote the union of all elements of $\mathscr{C}$.

Next we recall a few known results about unions of convex sets, which we reformulate for our needs.

Proposition $1[11,35]$. Let $\mathscr{C}$ be a complete family of nonempty closed convex sets lying strictly inside $D$. Then there exists a compact finite dimensional map $\sigma:|\mathscr{C}| \rightarrow|\mathscr{C}|$ such that $\sigma(C) \subset C$ for every $C \in \mathscr{C}$.

Let $\mathscr{C}$ be a finite family of nonempty closed convex sets lying strictly inside $D$, and let $s>\alpha(|\mathscr{C}|)$. Then there exists a finite complete family $\mathscr{C}^{\prime}$ such that

(I) $|\mathscr{C}|=\left|\mathscr{C}^{\prime}\right|$,

(II) $\operatorname{diam}_{k_{D}}\left(C^{\prime}\right) \leq s$ for each $c^{\prime} \in \mathscr{C}^{\prime}$,

(III) every $C^{\prime} \in \mathscr{C}^{\prime}$ is nonempty, closed, convex, and lies strictly inside $D$ (see (5)).

The above facts allow us to prove the following proposition.

Proposition 2. Let $C$ be a finite union of nonempty closed convex sets lying strictly inside $D$, and let $s>\alpha(C)$. Then there exists a finite dimensional map $\sigma: C \rightarrow C$ such that

(I') $k_{D}(x, \sigma(x)) \leq s$, each $x \in C$,

(II') $\sigma$ and the identity map are homotopic via the map $H: C \times[0,1] \rightarrow C$ defined by $H(x, t)=(1-t) \sigma(x)+t x$. 
It is known that for every finite union $C$ of nonempty closed convex sets lying strictly inside $D$, and every continuous $g: C \rightarrow C$, the Lefschetz number $\lambda(g)$ (with respect to the singular homology over the rationals) is well defined (see $[9,11]$ for details).

Lemma 2. Let $\mathscr{C}$ be a complete family of nonempty closed convex sets lying strictly inside $D$, and let $g:|\mathscr{C}| \rightarrow|\mathscr{C}|$ be continuous with $\lambda(g) \neq 0$. Then $g(C) \cap C \neq \varnothing$ for at least one $C \in \mathscr{C}$.

Proof. See [11].

Lemma 2 also yields the following fact.

Lemma 3. Let $C$ be a finite union of nonempty closed convex sets lying strictly inside $D$, and let $g: C \rightarrow C$ be continuous with $\lambda(g) \neq 0$. Then

$$
\inf _{x \in C} k_{D}(x, g(x)) \leq \alpha(C) \text {. }
$$

Now we are prepared to prove our first result.

Theorem 2. Let $C$ be a finite union of nonempty closed convex sets lying strictly inside $D$, and let $f: D \rightarrow D$ be holomorphic. If $C$ is $f$-invariant and $\lambda\left(f_{\mid C}\right) \neq$ 0 , then

$$
\inf _{i \in C}\|x-f(x)\|=0
$$

Proof. Let $\sigma: C \rightarrow C$ be as in Proposition 2. For $0 \leq t<1$ we define $f_{t}: C \rightarrow C$ by

$$
f_{t}(x)=(1-t) \sigma(f(x))+t f(x) .
$$

By Lemma 1, $f_{t}$ is an $\alpha$-contraction with constant $K$ given in (6), where $R=\operatorname{dist}(C, \partial D)$. Next we define a sequence of subsets $\left\{C_{n}\right\}$ of $C$ by setting

$$
C_{0}=C, \text { and } C_{n+1}=C_{n} \cap \operatorname{clco}\left(f_{t}\left(C_{n}\right)\right) .
$$

Each $C_{n}$ is a finite union of nonempty closed convex sets lying strictly inside $D$, and by (7)

$$
\alpha\left(C_{n}\right) \leq K \alpha\left(C_{n-1}\right)
$$

for $n=1,2, \ldots$. It is obvious that $f_{t}\left(C_{n}\right) \subset C_{n}$. Let $f_{t, n}$ denote the restriction of $f_{t}$ to $C_{n}$. By Proposition 2, $f_{t}$ and $f$ are homotopic, and therefore the equalities

$$
f_{t, n}=h \circ i, \quad f_{t, n-1}=i \circ h
$$

where $i: C_{n} \rightarrow C_{n-1}$ is an inclusion and $h: C_{n-1} \rightarrow C_{n}$ is the map induced by $f_{t}$, yield

$$
\lambda\left(f_{t, n}\right)=\lambda\left(f_{t, n-1}\right) \text { for } n=1,2, \ldots
$$

and

$$
\lambda\left(f_{t, n}\right)=\lambda\left(f_{\mid C}\right) \neq 0 \text { for } n=0,1, \ldots
$$

Hence by Lemma 3, we obtain

$$
\inf _{x \in C} k_{D}\left(x, f_{t}(x)\right)=0 .
$$


Now we are ready to achieve our aim. We have

$$
\begin{aligned}
\inf _{x \in C} k_{D}(x, f(x)) & \leq \inf _{x \in C}\left[k_{D}\left(x, f_{t}(x)\right)+k_{D}\left(f_{t}(x), f(x)\right)\right] \\
& \leq \inf _{x \in C}\left[k_{D}\left(x, f_{t}(x)\right)+L_{1}(\operatorname{clco} C)(1-t)\|\sigma(f(x))-f(x)\|\right] \\
& \leq L_{1}(\operatorname{clco} C)(1-t)(\operatorname{diam}(C))
\end{aligned}
$$

for all $t \in[0,1)\left(L_{1}(\right.$ clco $C)$ is defined in (4)). Hence

$$
0=\inf _{x \in C} k_{D}(x, f(x)) \geq \frac{L_{1}(\operatorname{clco} C)}{L_{2}(\operatorname{clco} C)} \inf _{x \in C}\|x-f(x)\| \geq 0
$$

$\left(L_{2}(\operatorname{clco} C)\right.$ is also defined in (4)), and the result follows.

As consequences of Theorem 2 we obtain the following two new results for holomorphic mappings.

Theorem 3. Let $C$ be a finite union of nonempty closed convex sets lying strictly inside $D$. If $C$ is contractible, $f: D \rightarrow D$ is holomorphic, and $C$ is $f$-invariant, then

$$
\inf _{x \in C}\|x-f(x)\|=0 .
$$

Proof. By the contractiblity of $C, \lambda\left(f_{\mid C}\right)=1$, and we may apply Theorem 2 .

Theorem 4. If $C_{1}, \ldots, C_{m}$ are nonempty closed convex sets lying strictly inside $D$ that satisfy condition (2), i.e., $C_{i} \cap C_{j} \neq \varnothing \Leftrightarrow|i-j| \leq 1$ for all $1 \leq i, j \leq m$, then for every holomorphic $f: D \rightarrow D$ such that $C=\bigcup_{i=1}^{m} C_{i}$ is $f$-invariant, we have inf $x \in C\|x-f(x)\|=0$.

Proof. $C$ is contractible [6].

\section{THE CASE $D=B^{n}$}

Let $H$ be a complex Hilbert space with scalar product $\langle\cdot, \cdot\rangle$ and norm $\|\cdot\|_{1}$, and let $B$ be the open unit ball of $H$. By $B^{n}$ we denote the Cartesian product of $n$ open unit balls. $B^{n}$ is, of course, the open unit ball in $H^{n}$ with the maximum norm $\|\cdot\|_{n}$. It is well known that in $B=B^{1}$ the Kobayashi distance is defined by

$$
k_{B}(x, y)=\arctan \left[(1-\sigma(x, y))^{1 / 2}\right],
$$

where

$$
\sigma(x, y)=\left(1-\|x\|_{1}^{2}\right)\left(1-\|y\|_{1}^{2}\right) /|1-\langle x, y\rangle|^{2}
$$

$[10,15,17,18,19,43]$. The Kobayashi distance on $B^{n}$ can be inductively defined by

$$
k_{B^{n}}\left(\left(x_{1}, x_{2}\right),\left(y_{1}, y_{2}\right)\right)=\max \left\{k_{B}\left(x_{1}, y_{1}\right), k_{B^{n-1}}\left(x_{2}, y_{2}\right)\right\}
$$

for $\left(x_{1}, x_{2}\right),\left(y_{1}, y_{2}\right) \in B \times B^{n-1}=B^{n}$. This distance coincides with the Carathéodory distance on $B^{n}$. (note that the Carathéodory distance and the Kobayashi distance are also equivalent on any open convex norm-bounded subset of $\mathbf{C}^{n}$ [34].)

Now we recall a few known properties of the Kobayashi distance $k_{B^{n}}$. The space $\left(B, k_{B}\right)$ has Opial's property $[15,17,36]$, i.e., for every $k_{B}$-bounded weakly convergent sequence $\left\{x_{j}\right\}$ in $B$ with $x=\mathrm{w}-\lim _{j} x_{j}$ and for every $y \in B$ different from $x$ the following inequality is valid:

$$
\liminf _{j} k_{B}\left(x, x_{j}\right)<\liminf _{j} k_{B}\left(y, x_{j}\right) \text {. }
$$


This implies that every sequence $\left\{x_{j}\right\}$ is a nonempty closed convex $k_{B^{n}}$ bounded subset $C$ of $B^{n}$ with $\operatorname{diam}_{k_{B^{n}}}(C)>0$ contains a subsequence $\left\{x_{j_{i}}\right\}$ for which there exist two points $x, y \in C$ such that $\lim _{i} k_{B^{n}}\left(x, x_{j_{i}}\right)$ and $\lim _{i} k_{B^{n}}\left(y, x_{j_{i}}\right)$ exist and are different. Since every ball in $\left(B, k_{B}\right)$ is uniformly convex with respect to linear convexity [26], we can apply the method of proof of the Belluce-Kirk-Steiner Theorem [3] to $\left(B^{n}, k_{B^{n}}\right)$ and obtain that $\left(B^{n}, k_{B^{n}}\right)$

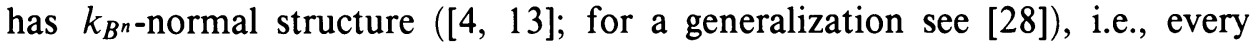
nonempty closed convex $k_{B^{n}}$-bounded subset $C$ of $B^{n}$ with $\operatorname{diam}_{k_{B^{n}}}(C)>0$ has a point $c \in C$ such that $\sup _{x \in C} k_{B^{n}}(x, c)<\operatorname{diam}_{k_{B^{n}}}(C)$. This implies that each $k_{B^{n}}$-nonexpansive self-mapping $f$ defined on a closed convex $k_{B^{n}}$ bounded subset $C$ of $B^{n}$ has a fixed point [25, 26, 29]. (For other properties of holomorphic and $k_{B^{n}}$-nonexpansive mappings in $B^{n}$ see $[10,12,14,15,17$, $18,19,24-27,30,38-41,43,44]$.) We now use Theorem 3 to extend this last result to nonconvex domains.

Theorem 5. Let $C$ be a finite union of nonempty, closed, convex, and $k_{B^{-}}$ bounded subsets of $B$. If $C$ is contractible, $f: B \rightarrow B$ is holomorphic, and $C$ is $f$-invariant, then $f$ has a fixed point in $C$.

Proof. By Theorem 3 there exists a sequence $\left\{x_{j}\right\}$ in $C$ such that

$$
\lim _{j} k_{B}\left(x_{j}, f\left(x_{j}\right)\right)=0 .
$$

Without loss of generality we may assume that $\left\{x_{j}\right\}$ converges weakly to $x \in C$. Opial's property now implies that $f(x)=x$.

Theorem 6. If $C_{1}, \ldots, C_{m}$ are nonempty, closed, convex, and $k_{B^{n}}$-bounded subsets of $B^{n}$ which satisfy (2), i.e., $C_{i} \cap C_{j} \neq \varnothing \Leftrightarrow|i-j| \leq 1$ for all $1 \leq i, j \leq$ $m$, then every holomorphic $f: B^{n} \rightarrow B^{n}$ such that $C=\bigcup_{i=1}^{m} C_{i}$ is $f$-invariant has a fixed point in $C$.

Proof. For $m=1$ see $[25,26,29]$. Applying mathematical induction (with respect to $m$ ), let $C=\bigcup_{i=1}^{m+1} C_{i}$ satisfy condition (2), and assume that $f: B^{n} \rightarrow$ $B^{n}$ is holomorphic an $C$ is $f$-invariant. Using arguments based on Zorn's Lemma we can find a minimal (with respect to inclusion) connected $f$-invariant set $E=\bigcup_{i=m_{1}}^{m_{2}} E_{i}$, where each $E_{i}$ is a nonempty weakly compact separable convex subset of $C_{i} \quad\left(m_{1} \leq i \leq m_{2}\right)$, and the number of the sets $E_{i}$ cannot be reduced. By the inductive hypothesis $E$ is a singleton as long as $m_{2}-m_{1}+1 \leq$ $m$. So we assume that $f$ is fixed point free and that $E=\bigcup_{i=1}^{m+1} E_{i}$. By Theorem 4 there is a sequence $\left\{x_{j}\right\}$ in $E$ such that $\lim _{j} k_{B^{n}}\left(x_{j}, f\left(x_{j}\right)\right)=0$. Without loss of generality (taking a subsequence if necessary) we can assume that all the $x_{j}$ are in one $E_{i_{0}}$. Hence there exist $y$ and $z$ in $E_{i_{0}}$ such that

$$
\lim _{j} k_{B^{n}}\left(y, x_{j}\right)<\lim _{j} k_{B^{n}}\left(z, x_{j}\right) \text {. }
$$

Recall that the asymptotic center of $\left\{x_{j}\right\}$ with respect to $E[1,8,13]$ is

$$
\mathrm{AC}\left(E,\left\{x_{j}\right\}\right)=\left\{x \in E: r\left(x,\left\{x_{j}\right\}\right)=r\left(E,\left\{x_{j}\right\}\right)\right\},
$$

where

$$
r\left(x,\left\{x_{j}\right\}\right)=\limsup k_{B^{n}}\left(x, x_{j}\right),
$$

and

$$
r\left(E,\left\{x_{j}\right\}\right)=\inf _{x \in E} r\left(x,\left\{x_{j}\right\}\right) .
$$


It is easy to observe that for each $i, E_{i} \cap \mathrm{AC}\left(E,\left\{x_{j}\right\}\right)$ is either empty or weakly compact and convex. Next by (8) we get $E_{i_{0}} \neq E_{i_{0}} \cap \mathrm{AC}\left(E,\left\{x_{j}\right\}\right)$, and therefore $\mathrm{AC}\left(E,\left\{x_{j}\right\}\right) \neq E$. Since $\mathrm{AC}\left(E,\left\{x_{j}\right\}\right)$ is nonempty and $f$-invariant, it must have at least two connected components. Now for $t \geq r\left(E,\left\{x_{j}\right\}\right)=t_{1}$ we define $A(t)$ by

$$
A(t)=\left\{x \in E: r\left(x,\left\{x_{j}\right\}\right) \leq t\right\} .
$$

The same reasons as before yield the following properties of the sets $A(t)$ for $t \geq t_{1}$ :

(1) $A\left(t_{1}\right)=\mathrm{AC}\left(E,\left\{x_{j}\right\}\right) \neq E$,

(2) For $t_{2}=\min \left\{t \geq t_{1}: A(t)=E\right\}$ we have $A\left(t_{2}\right)=E$ and $A(t) \neq E$ for $t_{1} \leq t<t_{2}$

(3) If there exists $x$ such that $x \in E_{i} \cap A(t)$ with $t_{1} \leq t<t_{2}$, then $E_{i} \subset{\operatorname{cl} \bigcup t_{1} \leq t<t_{2}} A(t)$. Therefore by (8) $E_{i_{0}} \subset \operatorname{cl}_{t_{1} \leq t<t_{2}} A(t)$,

(4) $f(A(t)) \subset A(t)$ for all $t \geq t_{1}$,

(5) There exist $t_{1} \leq t_{3}<t_{2}$ and $\bar{m} \geq 2$ such that for every $t_{3} \leq t<t_{2}$ $A(t)$ consists of exactly $\bar{m}$ components $A_{1}(t), \ldots, A_{\bar{m}}(t)$,

(6) $\left\{A_{1}(t), \ldots, A_{\bar{m}}(t)\right\}_{t_{3} \leq t<t_{2}}$ can be partitioned into $f$-cycles; i.e., $f\left(A_{1}(t)\right)$ $\subset A_{2}(t), \ldots, f\left(A_{m_{1}-1}(t)\right) \subset A_{m_{1}}(t), f\left(A_{m_{1}}(t)\right) \subset A_{1}(t), f\left(A_{m_{1}+1}(t)\right) \subset$ $A_{m_{1}+2}(t), \ldots$. (We can assume such an order; if not, we just renumber our sets.)

(7) We can assume, for example, that the point $y \in E_{i_{0}}$ from (8) is an element of $A_{1}(t)$ for $t_{3} \leq t<t_{2}$.

By (iii) and (vii) the whole sequence $\left\{x_{j}\right\}$ lies in $E_{i_{0}} \cap \mathrm{cl}\left(\bigcup_{t_{3} \leq t<t_{2}} A_{1}(t)\right)=E_{i_{0}}$. Hence we can find a sequence $\left\{y_{j}\right\}$ in $\bigcup_{t_{3} \leq t<t_{2}} A_{1}(t)$ satisfying

$$
\lim _{j} k_{B^{n}}\left(y_{j}, f\left(y_{j}\right)\right)=0 .
$$

But then we see that the $k_{B^{n}}$-distance between any two sets from the mutually disjoint connected set $\bigcup_{t_{3} \leq t<t_{2}} A_{1}(t), \bigcup_{t_{3} \leq t<t_{2}} A_{2}(t), \ldots, \bigcup_{t_{3} \leq t<t_{2}} A_{m_{1}}(t)$ equals 0 . Condition (2) now implies that $m_{1}=2$. Therefore we have

$$
\begin{aligned}
& f\left(\bigcup_{t_{3} \leq t<t_{2}} A_{1}(t)\right) \subset \bigcup_{t_{3} \leq t<t_{2}} A_{2}(t), \\
& f\left(\bigcup_{t_{3} \leq t<t_{2}} A_{2}(t)\right) \subset \bigcup_{t_{3} \leq t<t_{2}} A_{1}(t),
\end{aligned}
$$

and

$$
\widetilde{E}=\left(\mathrm{cl} \bigcup_{t_{3} \leq t<t_{2}} A_{1}(t)\right) \cap\left(\mathrm{cl} \bigcup_{t_{3} \leq t<t_{2}} A_{2}(t)\right)
$$

is a nonempty weakly compact convex $f$-invariant set. Hence $f$ has a fixed point in $\widetilde{E}$. This contradicts the assumption that $f$ is fixed point free. Therefore $f$ does have a fixed point and the proof is complete.

Since the proofs of Theorems 2-6 use only metric and topological properties of holomorphic mappings, the above theorems are, in fact, true for all $k_{D^{-}}$ $\left(k_{B^{n}}\right)$ nonexpansive self-mappings of $C$. 


\section{NoNEXPANSIVE MAPPINGS}

We begin this section with the definition of normal structure with respect to a topology $\tau$. Let $(X,\|\cdot\|)$ be a Banach space, $\tau$ a Hausdorff topology on $X$, and $C$ a nonempty convex subset of $X . C$ is said to have normal structure with respect to the topology $\tau$ ( $\tau$-NS) [28] if every nonempty $\tau$ compact (i.e., compact in the topology $\tau$ ) convex norm-bounded subset $C_{1}$ of $C$ which contains more than one point has a nondiametral point, i.e., a point $c \in C_{1}$ for which $\sup _{x \in C_{1}}\|c-x\|<\operatorname{diam} C_{1}$. The following result was proved in [28] (see also [13, 33, 37]).

Theorem 7 [28]. Let $C$ be a nonempty convex subset of a Banach space $(X,\|\cdot\|)$ with a Hausdorff topology $\tau$. If $\operatorname{diam} C>0, C$ has $\tau$-NS and the intersections of $C$ with closed balls are $\tau$-closed, then every norm-bounded sequence $\left\{x_{j}\right\}$ contains a subsequence $\left\{x_{j_{i}}\right\}$ for which there exist points $x, y \in C$ such that

$$
\lim _{i}\left\|x-x_{j_{i}}\right\| \neq \lim _{i}\left\|y-x_{j_{i}}\right\| .
$$

Now the method of proof of Theorem 6 (we replace Theorem 4 by the theorem of Furi and Martelli [11]) also leads to the following result.

Theorem 8. Let $(X,\|\cdot\|)$ be a Banach space with a Hausdorff topology $\tau$, and let $C_{1}, \ldots, C_{m}$ be $m$ nonempty, $\tau$-compact, convex and norm-bounded subsets of $X$ with $\tau$-NS. Assume that the asymptotic radius function $r\left(\cdot,\left\{x_{j}\right\}\right): C=$ $\bigcup_{i=1}^{m} C_{i} \rightarrow \mathbf{R}$ is $\tau$-l.s.c. (i.e., lower semicontinuous with respect to the topology $\tau)$. If $C_{1}, \ldots, C_{m}$ satisfy condition (2), then $C$ has the fixed point property for nonexpansive mappings $(F F P)$.

Remark. It is sufficient to assume in Theorem 8 that every sequence $\left\{x_{j}\right\}$ contains a subsequence for which the asymptotic radius function is $\tau$-l.s.c. (see $[20,28])$. For examples of topologies $\tau$ satisfying the hypothesis of Theorem 8 see [28]. For results related to Theorem 8 see [16, 23, 42, 45, 46].

We now prove a result on the structure of the fixed point set Fix $f=\{x \in$ $X: f(x)=x\}$ of a nonexpansive self-mapping $f: C \rightarrow C$.

Theorem 9. Let $(X,\|\cdot\|)$ be a strictly convex Banach space, and let $C_{1}, \ldots, C_{m}$ be $m$ nonempty weakly compact convex subsets of $X$ with normal structure (with respect to the weak topology). If $C_{1}, \ldots, C_{m}$ satisfy condition (2), and $f: C=\bigcup_{i=1}^{m} C_{i} \rightarrow C$ is nonexpansive, then Fix $f$ is of the following form: $E=\bigcup_{i=m_{1}}^{m_{2}} E_{i}$, where each $E_{i} \subset C_{i}$ is nonempty, weakly compact, and convex, and $E$ is connected (i.e., $E_{i} \cap E_{i+1} \neq \varnothing$ for $m_{1} \leq i \leq m_{2}-1$ ).

Proof. Consider the intrinsic metric $\rho: C \times C \rightarrow \mathbf{R}$ defined by

$$
\rho(x, y)=\inf \{d: d \text { is the length of a broken line in } C \text { joining } x \text { with } y\} .
$$

Then a norm-nonexpansive $f: C \rightarrow C$ is also $\rho$-nonexpansive. The strict convexity of $X$ implies that for every pair $x, y \in C$ there exists exactly one broken line realizing the $\rho$-distance between $x$ and $y$. If $x, y \in \operatorname{Fix} f$, then this line is $f$-invariant and therefore it consists of fixed points of $f$. Our theorem is a simple consequence of this fact. 
Theorem 9 is a generalization of the well-known theorem for convex subsets of strictly convex Banach spaces [5].

We conclude with a common fixed point theorem which is a consequence of Theorem 9.

Theorem 10. If $X$ and $C$ are as in Theorem 9, and $\left\{f_{\beta}\right\}$ is a family of commuting nonexpansive self-mappings of $C$, then $\bigcap_{\beta}$ Fix $f_{\beta} \neq \varnothing$.

\section{ACKNOWLEDGMENT}

This paper was prepared during a visit of the first author to the Department of Mathematics at the University of Southern California. He is grateful to his colleagues at USC for their hospitality.

\section{REFERENCES}

1. A. G. Aksoy and M. A. Khamsi, Nonstandard methods in fixed point theory, Springer-Verlag, New York and Berlin, 1990

2. J. Banaś and K. Goebel, Measures of noncompactness in Banach spaces, Dekker, New York, 1980.

3. L. P. Belluce, W. A. Kirk, and E.. Steiner, Normal structure in Banach spaces, Pacific J. Math. 26 (1968), 433-440.

4. M. S. Brodskii and D. P. Milman, On the center of a convex set, Dokl. Akad. Nauk SSSR 59 (1948), 837-840.

5. R. E. DeMarr, Common fixed points for commuting contraction mappings, Pacific J. Math. 13 (1963), 1139-1141.

6. J. Dugundji, Topology, Allyn and Bacon, Boston, MA, 1966.

7. C. J. Earle and R. S. Hamilton, A fixed point theorem for holomorphic mappings, Proc. Sympos. Pure Math., vol. 16, Amer. Math. Soc., Providence, RI, 1970, pp. 61-65.

8. M. Edelstein, The construction of an asymptotic center with a fixed point property, Bull. Amer. Math. Soc. 78 (1972), 206-208.

9. S. Eilenberg and N. Steenrod, Foundations of algebraic topology, Princeton Univ. Press, Princeton, NJ, 1952.

10. T. Franzoni and E. Vesentini, Holomorphic maps and invariant distances, North-Holland, Amsterdam, 1980.

11. M. Furi and M. Martelli, A Lefschetz type theorem for the minimal displacement of points under maps defined on a class of ANR's, Boll. Un. Mat. Ital. 10 (1974), 174-181.

12. K. Goebel, Fixed points and invariant domains of holomorphic mappings of Hilbert ball, Nonlinear Anal. 6 (1982), 1327-1334.

13. K. Goebel and W. A. Kirk, Topics in metric fixed point theory, Cambridge Univ. Press, Cambridge, 1990.

14. K. Goebel and S. Reich, Iterating holomorphic self-mappings of the Hilbert ball, Proc. Japan Acad. 58 (1982), 349-352.

15. __ Uniform convexity, hyperbolic geometry and nonexpansive mappings, Dekker, New York, 1984.

16. K. Goebel and R. Schöneberg, Moons, bridges, birds, ..., and nonexpansive mappings in Hilbert space, Bull. Austral. Math. Soc. 17 (1977), 463-466.

17. K. Goebel, T. Sẹkowski, and A. Stachura, Uniform convexity of hyperbolic metric and fixed points of holomrphic mappings in the Hilbert ball, Nonlinear Anal. 4 (1980), 1011-1021.

18. K. T. Hahn, Geometry on the unit ball of a complex Hilbert space, Canad. J. Math. 30 (1978), 22-31. 
19. L. A. Harris, Schwarz-Pick systems of pseudometrics for domains in normed linear spaces, Advances in Holomorphy (J. A. Barosso, ed.), North-Holland, Amsterdam, 1979, pp. 345406.

20. M. S. Khamsi, On the weak*-fixed point property, Contemp. Math., vol. 85, Amer. Math. Soc., Providence, R.I., 1989, pp. 325-337.

21. W. A. Kirk, A fixed point theorem for mappings which do not increase distances, Amer. Math. Monthly 72 (1965), 1004-1006.

22. S. Kobayashi, Hyperbolic manifolds and holomorphic mappings, Dekker, New York, 1970.

23. Cl. Krauthausen, G. Müler, J. Reinermann, and R. Schöneberg, New fixed point theorems for compact and nonexpansive mappings and applications to Hammerstein equations, Sonderforschungsbereich 72 an der Universität Bonn, 1975.

24. T. Kuczumow, Nonexpansive retracts and fixed points of nonexpansive mappings in the Cartesian product of $n$ Hilbert balls, Nonlinear Anal. 9 (1985), 601-604.

25. __, Nonexpansive mappings and isometries of Hilbert $n$-balls with hyperbolic metrics, Rozprawy Wydziału Mat.-Fiz.-Chem., Rozprawy Habilt. 44, Lublin, 1987.

26. $\ldots$, Fixed points of holomorphic mappings in the Hilbert ball, Colloq. Math. 55 (1988), 101-107.

27. T. Kuczumow and W. O. Ray, Isometries in the Cartesian product of $n$ unit open Hilbert balls with a hyperbolic metric, Ann. Mat. Pura Appl. 152 (1988), 359-374.

28. T. Kuczumow, S. Reich, M. Schmidt, and A. Stachura, Strong asymptotic normal structure and fixed points in product spaces, preprint.

29. T. Kuczumow and A. Stachura, Convexity and fixed points of holomorphic mappings in Hilbert ball and polydisc, Bull. Polish Acad. Sci. 34 (1986), 189-193.

30. Fixed points of holomorphic mappings in the Cartesian product of $n$ unit Hilbert balls, Canad. Math. Bull. 29 (1986), 281-286.

31. __ Iterates of holomorphic and $k_{D}$-nonexpansive mappings in convex domains in $\mathbf{C}^{n}$, Adv. Math. 81 (1990), 90-98.

32. K. Kuratowski, Sur les éspaces complets, Fund. Math. 15 (1930), 301-309.

33. T. Landes, Permanence properties of normal structure, Pacific J. Math. 110 (1984), 123-143.

34. L. Lempert, Holomorphic retracts and intrinsic metrics in convex domains, Anal. Math. 8 (1982), 257-261.

35. R. D. Nussbaum, The fixed point index for local condensing maps, Ann. Mat. Pura Appl. 89 (1971), 217-258.

36. Z. Opial, Weak convergence of the sequence of successive approximations for nonexpansive mappings, Bull. Amer. Math. Soc. 73 (1967), 591-597.

37. J. Penot, Fixed point theorems without convexity, Bull. Soc. Math. France Mem. 60 (1979), 129-152.

38. S. Reich, Averaged mappings in the Hilbert balls, J. Math. Anal. Appl. 109 (1985), 199-206.

39. __ Nonlinear semigroups, holomorphic mappings, and integral equations, Proc. Sympos. Pure Math., vol. 45, part 2, Amer. Math. Soc., Providence, RI, 1986, pp. 307-324.

40. ___ Approximating fixed points of holomorphic mappings, CAMS Report \#91-4, Math. Japonica (to appear).

41. S. Reich and I. Shafrir, Nonexpansive iterations in hyperbolic spaces, Nonlinear Anal. 15 (1990), 537-558.

42. j. Reinermann and R. Schöneberg, Some results and problems in the fixed point theory for nonexpansive and pseudocontractive mappings in Hilbert space, Fixed Point Theory and Its Applications (S. Swaminathan, ed.), Academic Press, New York, 1976, pp. 187-196.

43. W. Rudin, Function theory in the unit ball of $\mathbf{C}^{n}$, Springer-Verlag, New York and Berlin, 1980.

44. I. Shafrir, Common fixed points of commuting holomorphic mappings in the product of $n$ Hilbert balls, preprint. 
45. R. Smarzewski, On firmly nonexpansive mappings, Proc. Amer. Math. Soc. 113 (1991), 723-725.

46. A. Stachura, Fixed points of nonexpansive mappings in the sum of two sets, preprint.

Department of Mathematics, Lublin Technical University, 20-618 Lublin, Poland

Center for applied Mathematical Sciences, Department of Mathematics, University of Southern California, Los ANGeles, California 90089-1113

InStytut Matematyki UMCS, 20-031 Lublin, Poland 